\title{
Patients with inferior Ml: right ventricular involvement
}

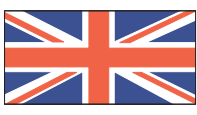

\author{
Mel Humphreys, MA, BSc (Hons), ENB 124, RGN, ONC, Senior Lecturer, Postregistration Studies, \\ School of Health, University of Wolverhampton, UK
}

Senior Lecturer, School of Health, The Chestnuts, New Cross Hospital, Wolverhampton WV10 0QP, UK.

e-mail: in5277@wlv.ac.uk

\section{INTRODUCTION}

Coronary heart disease is one of the biggest killers in the UK. More than 1.4 million people suffer from angina; around 300,000 have heart attacks every year; and more than 110,000 die of heart problems in England every year. It remains one of the most common causes of death in the world (Department of Health, 2000).

The individual with an acute myocardial infarction (MI) has experienced a life-threatening event and must begin to adjust to many lifestyle changes. It has been reported that as many as $40 \%$ of all patients with an inferior wall MI also suffer a right ventricular MI (RVI) (Ambrose, 1997). This presents a major clinical challenge to the nurse working within an acute care area.

It is important to note that nearly $50 \%$ of patients suffering an inferior MI will have complications or distinguishing features associated with an increased mortality that will substantially alter an otherwise favourable prognosis (Berger and Ryan, 1990).

Early accurate diagnosis and appropriate management are of fundamental importance in order to enhance long-term prognosis.

\section{ECG IDENTIFICATION OF RVI}

Myocardial ischaemia, injury and necrosis are reflected on electrocardiograms (ECGs) by disturbances in depolarisation and repolarisation, which appear in the QRS, ST-segment and $\mathrm{T}$-wave portions. Disorders of depolarisation and repolarisation may be confined to only one segment of the myocardium; therefore, multiple leads are necessary to provide a comprehensive view of these electrical events throughout the heart (Box 1).

In the case of inferior MIs and their high association with right ventricular involvement (Zehender et al., 1993), a rightsided ECG should be recorded as early as possible in order to provide an overview of the right ventricle as well as the left ventricle. Risk stratification is well worthwhile. Studies have indicated that resolution of ECG changes over the right ventricle can occur within 10-24 hours of the onset of symptoms (Braat et al., 1983; Thompson, 1997). This provides a rationale as to why subsequent right-sided ECG recordings are not routinely practised within acute care areas.

Identifying a RVI is difficult without information from the right precordial leads. A RVI can lead to right ventricular failure or right bundle-branch block. The classic changes are STsegment elevation, pathological $\mathrm{Q}$ waves, and inverted $\mathrm{T}$ waves in the right precordial leads V2R to V4R, with V4R offering the most reliability (Berger and Thomas, 1990). These findings may be diagnostic even in the absence of clinically evident haemodynamic disturbance of right ventricular infarction.

If these leads are not available, you can observe leads II, III, and $\mathrm{aVf}$ (to diagnose inferior involvement) and observe leads V1 and V2 for elevated ST segments - these are considered to be subtle indicators of RVI (Ambrose, 1997). Importantly, and in this circumstance, accurate haemodynamic monitoring plays an important role in the subsequent risk identification.

\section{PHYSIOLOGY OF RVI}

Typically, RVI occurs when there is an occlusion of the right coronary artery proximal to the acute marginal branches. However, it may also occur with an occlusion of the left circumflex artery in patients who have left-dominant coronary circulation. Although less common, occlusion of the left anterior descending artery may also result in infarction of the anterior right ventricle (Kinch and Ryan, 1994).

Branches of the right coronary artery are also responsible for the supply of blood and oxygen to the intraventricular septum and the inferior surface of the left ventricle. Concomitant damage is, therefore, nearly always present in these two areas (Roberts and Murmor, 1983). The right coronary artery is the dominant supplier of blood to the sino-atrial node, and diminished blood supply can cause bradyarrhythmias. RVI dysfunction of the necrosed right ventricle gives rise to a picture of right-sided heart failure. The impaired right ventricle is unable to pump blood effectively into the left atrium, causing a reduction in preload to the left ventricle and a subsequent decline in cardiac output. This can be manifested by hypotension, elevated jugular venous pressure, a positive Kussmaul's venous sign and clear lung fields. Peripheral shutdown may also be evident.

A common error in the management of RVI in the past has been to attribute the elevation of venous pressure to transmitted left heart pressures and left ventricular dysfunction (Thompson, 1997). Therapy with diuretics and nitrates in an RVI further compounds this problem, resulting in a sharp reduction in left ventricular preload. The cardiac output would continue to decline and death could occur from cardiogenic shock (Horan and Flowers, 1999). Indeed, hypotension following administration of sublingual nitrate should immediately raise the suspicion 


\section{BOX 1}

ECG showing sinus rhythm with an inferior wall myocardial infarction showing right ventricular involvement

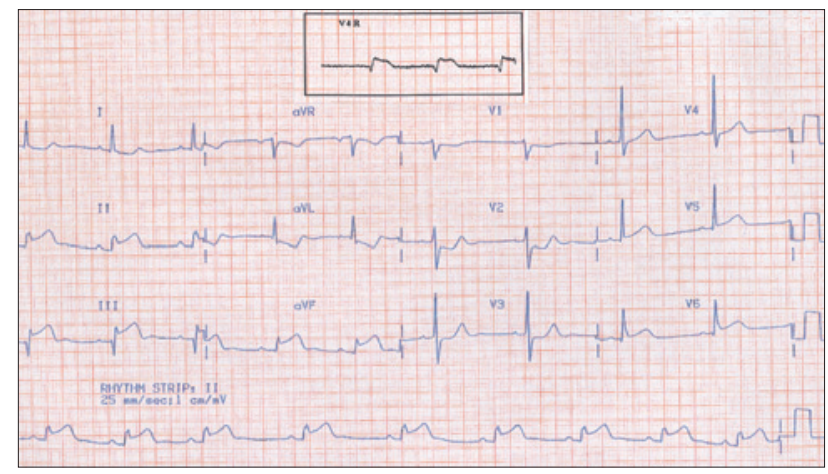

\section{COMMENTARY}

This 12-lead ECG shows the following features:

Heart rate, $55 \mathrm{bpm}$

- Rhythm, regular

- P waves, normal

- PR interval, $0.14 \mathrm{~s}$

QT interval, $0.44 \mathrm{~s}$ (normal)

- QRS axis, normal

- ST segment elevation in leads II, III and aVf, suggesting inferior MI

- ST segment elevation in lead V4R, suggesting an RVI (recorded: right fifth intercostal space, mid-clavicular line)

- Reciprocal ST depression in the anterior lead V2.

of right ventricular involvement (Berger and Thomas, 1990). Kinch and Ryan (1994) estimate that up to half of inferior MIs are complicated in this way. Chest X-ray, ECG and echocardiographic data can assist in determining the need for diuretics or vasodilators.

Where there is an established RVI associated with low cardiac output, volume loading with normal saline or volume expanding colloid often resolves hypotension and improves cardiac output (Kinch and Ryan, 1994). However, the haemodynamic response can be variable, and pulmonary oedema may be provoked. It is, therefore, imperative that astute physiological cardiac output measurements and rhythm monitoring are continued.

Episodes of junctional rhythm (Box 2) (and or idioventricular rhythm) are common arrhythmias following inferior MI, due to the alterations in blood flow to the septum and consequently sinus node and atrioventricular node. These are usually intermittent and require no treatment (Nieman and Smith, 1999). However, the consequences in association with a RVI cause more concern. It is well established that about $70 \%$ of the blood flows passively from the atria into the ventricles; however, contraction of the atria forces the remaining blood into the ventricles. The final push accounts for about $30 \%$ of the blood passed into the ventricles (Tortora and Anagnostakos, 2000). The resultant haemodynamic consequences only serve to compromise the patient's haemodynamic stability further. For this reason atrioventricular synchrony and the sustaining of adequate right ventricular preload are important factors in the care of these patients (Kinch and Ryan, 1994). It may be necessary for temporary atrioventricular pacing to be undertaken.

\section{BOX 2}

ECG showing features associated with junctional rhythm

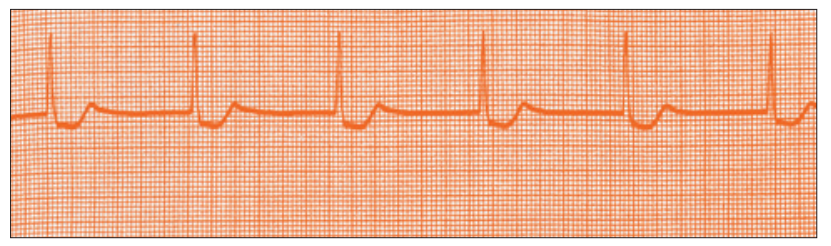

\section{COMMENTARY}

Single-lead ECG showing the following features:

Heart rate, $50 \mathrm{bpm}$

- Rhythm, regular

- P waves, absent (hidden in QRS complexes)

- QRS duration, 0.06-0.08 s

- ST segment depression is present.

\section{SUMMARY}

Involvement of the right ventricle in inferior wall infarction is common and presents a major challenge to the nurse. Clinically apparent haemodynamic consequences are observed in $20-25 \%$ of patients, and the risk of in-hospital mortality is seven to eight times that for patients with isolated acute inferior wall MIs (Wellens, 1993). The in-hospital mortality rate of $31 \%$ and high incidence of severe complications such as cardiogenic shock, ventricular fibrillation and myocardial rupture, emphasise the importance of the application of specialised cardiac knowledge to this presentation (Zehender et al., 1993), despite early thrombolysis (Giannitsis et al., 1997). However, by day 7, survivors of right ventricular MIs generally have normal right ventricular function restored, and thus have an identical prognosis to other uncomplicated MIs. It is for these reasons that physiological measurements, including regular and meticulous examination of the jugular venous pulse, should be a routine part of management of inferior MI to allow early detection of right ventricular involvement.

\section{REFERENCES}

Ambrose ML, Mauro E, Nash J, Sinovic D. (1997). ECG Interpretation made Incredibly Easy! Pennsylvania: Springhouse.

Berger PB, Thomas JR. (1990). Inferior myocardial infarction: high-risk subgroups. Circulation; 81: 401-411.

Bratt SH, Brugada P, de Zwaan C. (1983). Value of ECG in diagnosing right ventricular involvement in patients with an acute inferior wall MI. British Heart Journal; 49: 368-372.

Department of Health. (2000). National Service Framework for Coronary Heart Disease. Modern Standards and Service Models. London: The Stationery Office.

Giannitsis E, Potratz J, Wiegand U, Stierle U, Djonlagic H, Sheikhzadeh A. (1997). Impact of early accelerated dose tissue plasminogen activator on in-hospital patency of infarcted vessel in patients with acute right ventricular infarction. Heart; 77: 512-516.

Horan LG, Flowers NC. (1999). Right ventricular infarction: specific requirements of management. American Family Physician; 60: 1727-1734.

Kinch JW, Ryan TJ. (1994). Right ventricular infarction. New England Journal of Medicine; 330: 1211-1217.

Nieman W, Smith MJ. (1999). Arrhythmias post-MI. American Journal of Nursing; (1 May), www.nursingcenter.com/journals/ (Updated 5 May 1999).

Roberts R, Murmor AT. (1983). Right ventricular infarction. Annals of Revised Medicine; 34: 337-338.

Thompson PL. (1997). Coronary Care Manual. London: Churchill Livingstone.

Tortora GJ, Anagnostakos NP. (2000). Principles of Anatomy and Physiology (7th edition). New York: Harper Collins.

Wellens HJ. (1993). Right ventricular infarction. New England Journal of Medicine; 328: 1036-1038.

Zehender M, Kasper W, Kauder E. (1993). Right ventricular infarction as an independent predictor of prognosis after acute inferior myocardial infarction. New England Journal of Medicine; 328: 981-988. 